\title{
Convergence to Travelling Fronts of Solutions of the p-System with Viscosity in the Presence of a Boundary
}

\author{
Akitaka Matsumura \& Ming Mei
}

Communicated by T.-P. LIU

Dedicated to Professor SEIJI UKAI on his 60th birthday

\begin{abstract}
We study the asymptotic behavior as time goes to infinity of solutions to the initial-boundary-value problem on the half space $R_{+}$for a one-dimensional model system for the isentropic flow of a compressible viscous gas, the so-called $p$-system with viscosity. As boundary conditions, we prescribe the constant state at infinity and require that the velocity be zero at the boundary $x=0$. When the velocity at infinity is negative and satisfies a condition on the magnitude, we prove that if the initial data are suitably close to those for the corresponding outgoing viscous shock profile, which is suitably far from the boundary, then a unique solution exists globally in time and tends toward the properly shifted viscous shock profile as the time goes to infinity. The proof is given by an elementary energy method.
\end{abstract}

\section{Contents}

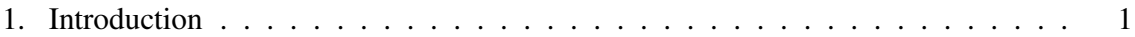

2. Preliminaries and Main Theorem . . . . . . . . . . . . . . . . 4

2.1. Viscous Shock Profile . . . . . . . . . . . . . . . . . . . 4

2.2. Location of the Shift . . . . . . . . . . . . . . . 5

2.3. Main Result . . . . . . . . . . . . . . . . . . . . . . 7

3. Reformulation of the Original Problem . . . . . . . . . . . . . . 8

4. Proof of the a Priori Estimates . . . . . . . . . . . . . . . 12

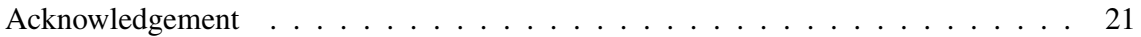

References . . . . . . . . . . . . . . . . . . . . . . . . . 21

\section{Introduction}

We consider a one-dimensional model system for the isentropic flow of a compressible viscous gas, the so-called $p$-system with viscosity, on the half space 
$R_{+}=(0, \infty)$ in the form

$$
\left.\begin{array}{rl}
v_{t}-u_{x} & =0 \\
u_{t}+p(v)_{x} & =\left(\frac{\mu u_{x}}{v}\right)_{x} \\
p(v) & =a v^{-\gamma}
\end{array}\right\} \quad(x, t) \in R_{+} \times R_{+},
$$

with the initial and boundary conditions

$$
\begin{gathered}
(v, u)(x, 0)=\left(v_{0}, u_{0}\right)(x), \quad x \in R_{+}, \\
u(0, t)=0, \quad(v, u)(+\infty, t)=\left(v_{+}, u_{+}\right), \quad t \in R_{+} .
\end{gathered}
$$

Here, $v(>0)$ is the specific volume, $u$ is the velocity, $\mu(>0)$ is the constant viscosity, $p(v)=a v^{-\gamma}$ is the pressure, $\gamma \geqq 1$ is the adiabatic constant, and $a$ is a positive gas constant. We are especially interested in the asymptotic behavior of the solution as the time goes to infinity, and how it is influenced by the prescribed constant state $\left(v_{+}, u_{+}\right)$at infinity. Physically, we expect the asymptotic behavior essentially to depend only on the sign of the velocity $u_{+}$at infinity. In particular, the solution is expected to tend toward an outgoing viscous shock profile (a front) if $u_{+}<0$, and toward an outgoing rarefaction wave if $u_{+}>0$. Since the case $u_{+}>0$ was recently solved in a very satisfactory fashion by Matsumura \& NishinaRA [13], we study the case $u_{+}<0$ here. The viscous shock profile with a shock speed $s$ is a travelling-wave solution of (1.1) on the whole space $R=(-\infty, \infty)$ of the form $(v, u)=(V, U)(\xi)(\xi=x-s t)$, satisfying the condition $(V, U)( \pm \infty)=$ $\left(v_{ \pm}, u_{ \pm}\right)$. It is well known that such a viscous shock profile exists and is unique up to shift, under the Rankine-Hugoniot (R-H) conditions

$$
\begin{aligned}
-s\left(v_{+}-v_{-}\right)-\left(u_{+}-u_{-}\right) & =0, \\
-s\left(u_{+}-u_{-}\right)+\left(p\left(v_{+}\right)-p\left(v_{-}\right)\right) & =0
\end{aligned}
$$

and the entropy condition

$$
u_{+}<u_{-} .
$$

In our present problem, the desired viscous shock profile is to be determined, so that for any given $\left(v_{+}, u_{+}\right)$with $v_{+}>0, u_{+}<0$ and with $u_{-}=0$, the values of $v_{-}>0$ and $s>0$ are uniquely given by the R-H condition (1.4):

$$
a v_{+}^{-\gamma+1}\left(1-\left(\frac{v_{-}}{v_{+}}\right)^{-\gamma}\right)\left(1-\frac{v_{-}}{v_{+}}\right)=-u_{+}^{2}, \quad s=\frac{-u_{+}}{v_{+}-v_{-}},
$$

with $v_{-}<v_{+}$. The aim of this paper is to discuss the asymptotic convergence of a solution toward a shift of this viscous shock profile.

The stability of viscous shock profiles for the Cauchy problem for various systems has been studied in many works; see $[1,2,4-6,10-12,14-16,18]$ and the references therein. From both the mathematical and physical point of view it is natural to investigate next the asymptotic behavior of solutions in the presence of boundaries. For the Burgers equation on the half space $R_{+}$with a Dirichlet boundary condition, the first treatment of the asymptotic convergence toward the viscous 
shock profile was given by LiU \& YU [9] (see also Yu [19]) by the method of pointwise estimates. Recently, the problem for the generalized Burgers equation has been thoroughly analyzed by LIU \& NISHIHARA [8] by the elementary weighted energy method. The asymptotic convergence toward the corresponding rarefaction wave for the scalar conservation law with viscosity and with a Dirichlet boundary has been studied by LiU, Matsumura \& Nishinara [7], while the $p$-system with viscosity has been very recently analyzed by MATSUMURA \& NisHiHARA [13] and PAN, LiU \& NishiHara [17]. However, there are no works on the asymptotic convergence to the viscous shock profile for physically meaningful systems with the boundary effect.

In each of the previous cases, determining the amount of shift of the asymptotic viscous shock profile plays an important role in the treatment of stability. Even for the scalar cases, LiU \& YU [9] and LiU \& Nishinara [8] needed a difficult analysis to locate the shift, since it cannot be determined explicitly because of the viscosity term. So we had thought that the case of the system is much more difficult in many aspects. However, it turns out that the $p$-system with viscosity on the half space has several features better than those for the scalar case with boundary and also for systems without boundary:

1. Since there are no standing waves for the $p$-system, any waves with negative speed are expected to be reflected at the boundary and finally be captured by the outgoing shock profile. This makes the variations of asymptotic behavior of the solution simpler than that for the Cauchy problem case, that is, we may classify the behaviour in terms of the sign of $u_{+}$.

2. The $p$-system with viscosity is not uniformly parabolic, i.e., there is no viscosity term for the specific volume $v(x, t)$ and we cannot impose the boundary value of $v(x, t)$, which usually gives difficulties. However, this is really the reason why we can specify the shift $\alpha$ of the outgoing viscous shock profile $V(x-s t+\alpha)$ explicitly by the equation for $v(x, t)$, and we can expect that the value of $v(x, t)$ on the boundary is automatically controlled by the effects of boundary and viscosity, so that the velocity $u(x, t)$ tends to $U(x-s t+\alpha)$ with the same shift $\alpha$. The details will be discussed in the following section.

In this paper, under these considerations, we shall show that when $u_{+}<0$, there exist a viscous shock profile $(V, U)(x-s t)$ unique up to a shift, and that if the viscous shock profile is suitably far from the boundary and if the initial perturbation is suitably small, then the global solution of the initial-boundary-value problem (1.1)-(1.3) exists, is unique, and tends toward the shifted viscous shock profile $(V, U)(x-s t+\alpha)$, where $\alpha$ is a constant uniquely determined by the initial data and the viscous shock profile.

This paper is organized as follows. After some notations are given below, the properties of the viscous shock profiles, a heuristic argument on how the shift $\alpha$ is determined, and the main theorem are stated in Section 2. In Section 3, we reformulate the original problem to obtain a new initial-boundary-value problem, and prove the global existence and the asymptotic behavior of the solution for the reformulated initial-boundary-value problem by proving local existence together 
with a priori estimates. The proof of the a priori estimates by the energy method will be given in Section 4.

Notation. $L^{2}$ denotes the space of measurable functions on $R_{+}$which are square integrable, with the norm

$$
\|f\|=\left(\int_{0}^{\infty}|f(x)|^{2} d x\right)^{1 / 2} .
$$

$H^{l}(l \geqq 0)$ denotes the Sobolev space of $L^{2}$-functions $f$ on $R_{+}$whose derivatives $\partial_{x}^{j} f, j=1, \ldots, l$, are also $L^{2}$-functions, with the norm

$$
\|f\|_{l}=\left(\sum_{j=0}^{l}\left\|\partial_{x}^{j} f\right\|^{2}\right)^{1 / 2} .
$$

Let $T$ be a positive constant and let $B$ be a Banach space. $C^{k}(0, T ; B)(k \geqq 0)$ denotes the space of $B$-valued $k$-times continuously differentiable functions on $[0, T]$, and $L^{2}(0, T ; B)$ denotes the space of $B$-valued $L^{2}$-functions on $[0, T]$. The corresponding spaces of $B$-valued functions on $[0, \infty)$ are defined similarly. In what follows, $C$ denote generic positive constants.

\section{Preliminaries and Main Theorem}

\subsection{Viscous Shock Profile}

We first recall the properties of viscous shock profiles. Viscous shock profiles are the travelling-wave solutions of (1.1) on the whole space of the form

$$
(v, u)(x, t)=(V, U)(\xi), \quad \xi=x-s t,
$$

which must satisfy

$$
-s V^{\prime}-U^{\prime}=0, \quad-s U^{\prime}+p(V)^{\prime}=\mu\left(\frac{U^{\prime}}{V}\right)^{\prime}, \quad(V, U)( \pm \infty)=\left(v_{ \pm}, u_{ \pm}\right),
$$

where ${ }^{\prime}=d / d \xi, s$ is the shock speed and $\left(v_{ \pm}, u_{ \pm}\right)$are the given constant states at $\xi= \pm \infty$. Integrating (2.2) under the Rankine-Hugoniot condition (1.4), we reduce problem (2.2) to

$$
\begin{gathered}
\frac{\mu s V^{\prime}}{V}=-s^{2} V-p(V)-b \equiv: h(V), \quad V( \pm \infty)=v_{ \pm}, \\
U=-s\left(V-v_{ \pm}\right)-u_{ \pm},
\end{gathered}
$$

where $b=-s^{2} v_{ \pm}-p\left(v_{ \pm}\right)$. In this paper, we are interested in the case $u_{+}<u_{-}=$ 0 , and in the 2-shock, i.e., the front shock, $s>0$. In this case, as mentioned in Section 1 , for any given $\left(v_{+}, u_{+}\right)\left(u_{+}<0, v_{+}>0\right)$, we know that $v_{-}\left(0<v_{-}<v_{+}\right)$and $s>0$ are uniquely determined by the R-H condition (1.4); see also (1.6). Then, in view of the convexity of $p(v)$, i.e., $p^{\prime \prime}(v)>0$ for $v>0$, the standard arguments 
for the ordinary differential equations assert the existence of a solution $(V, U)(\xi)$ of (2.2) satisfying

Proposition 2.1. For any $\left(v_{+}, u_{+}\right)\left(v_{+}>0=u_{-}>u_{+}\right)$, there exist unique $v_{-}\left(v_{+}>v_{-}>0\right)$ and $s(>0)$ satisfying (1.6), and a viscous shock profile $(V, U)(\xi)(\xi=x-s t)$ unique up to a shift, which connects $\left(v_{-}, 0\right)$ and $\left(v_{+}, u_{+}\right)$, satisfying

$$
\begin{gathered}
0<v_{-}<V(\xi)<v_{+}, \quad u_{+}<U(\xi)<0 \\
h(V)>0, \quad V_{\xi}=V h(V) / s \mu>0 \\
\left|V(\xi)-v_{ \pm}\right|=O(1)\left|v_{+}-v_{-}\right| e^{-c_{ \pm}|\xi|} \\
\left|U(\xi)-u_{ \pm}\right|=O(1)\left|v_{+}-v_{-}\right| e^{-c_{ \pm}|\xi|}
\end{gathered}
$$

as $\xi \rightarrow \pm \infty$, where $c_{ \pm}=v_{ \pm}\left|p^{\prime}\left(v_{ \pm}\right)+s^{2}\right| / \mu s>0$.

\subsection{Location of the Shift}

We first fix the viscous shock profile $(V, U)(x-s t)$ mentioned above. We consider the situation where the initial data $\left(v_{0}, u_{0}\right)(x)$ are given in a neighborhood of $(V, U)(x-\beta)$ for some constant $\beta>0$, so that we can describe how $(V, U)(x-$ $\beta$ ) is away from the boundary by taking $\beta$ large. Then, we make a heuristic argument to determine which of the shifted profiles $(V, U)(x-s t+\alpha-\beta)$ the solution tends toward. From the first equation of (1.1), we have

$$
(v-V)_{t}=(u-U)_{x},
$$

where $(V, U)=(V, U)(x-s t+\alpha-\beta)$. Integrating (2.6) over $[0,+\infty)$ with respect to $x$ and using the boundary condition (1.3) yields

$$
\frac{d}{d t} \int_{0}^{\infty}[v(x, t)-V(x-s t+\alpha-\beta)] d x=\left.(u-U)\right|_{x=0} ^{\infty}=U(-s t+\alpha-\beta) .
$$

Integrating (2.7) again with respect to $t$, we have

$$
\begin{aligned}
& \int_{0}^{\infty} {[v(x, t)-V(x-s t+\alpha-\beta)] d x } \\
& \quad=\int_{0}^{\infty}\left[v_{0}(x)-V(x+\alpha-\beta)\right] d x+\int_{0}^{t} U(-s \tau+\alpha-\beta) d \tau .
\end{aligned}
$$

If we suppose that $v(x, t)$ tends to $V(x-s t+\alpha-\beta)$ in $L^{1}$ as $t \rightarrow \infty$, the right-hand side of (2.8) must go to zero as $t \rightarrow \infty$. Hence, if we set

$$
I(\alpha):=\int_{0}^{\infty}\left[v_{0}(x)-V(x+\alpha-\beta)\right] d x+\int_{0}^{\infty} U(-s t+\alpha-\beta) d t,
$$


the shift $\alpha$ must be determined so that $I(\alpha)=0$. Differentiating $I(\alpha)$ with respect to $\alpha$ gives

$$
\begin{aligned}
I^{\prime}(\alpha) & =-\int_{0}^{\infty} V^{\prime}(x+\alpha-\beta) d x+\int_{0}^{\infty} U^{\prime}(-s t+\alpha-\beta) d t \\
& =-v_{+}+V(\alpha-\beta)+\frac{1}{s} U(\alpha-\beta) \\
& =-v_{+}+\frac{1}{s}\left(s v_{-}+u_{-}\right) \\
& =v_{-}-v_{+},
\end{aligned}
$$

where we used formula (2.3). Hence, it follows that

$$
I(\alpha)=\int_{0}^{\infty}\left[v_{0}(x)-V(x-\beta)\right] d x+\int_{0}^{\infty} U(-s t-\beta) d t+\left(v_{-}-v_{+}\right) \alpha .
$$

Thus, the shift $\alpha=\alpha(\beta)$ is determined explicitly by

$$
\alpha:=\frac{1}{v_{+}-v_{-}}\left\{\int_{0}^{\infty}\left[v_{0}(x)-V(x-\beta)\right] d x+\int_{0}^{\infty} U(-s t-\beta) d t\right\},
$$

and it follows from $(2.8)$ and $I(\alpha)=0$ that

$$
\begin{aligned}
\int_{0}^{\infty} & {[v(x, t)-V(x-s t+\alpha-\beta)] d x } \\
& =\int_{0}^{\infty}\left[v_{0}(x)-V(x+\alpha-\beta)\right] d x+\int_{0}^{t} U(-s \tau+\alpha-\beta) d \tau \\
& =I(\alpha)-\int_{t}^{\infty} U(-s \tau+\alpha-\beta) d \tau \\
& =-\int_{t}^{\infty} U(-s \tau+\alpha-\beta) d \tau \rightarrow 0 \quad \text { as } t \rightarrow \infty .
\end{aligned}
$$

In particular,

$$
\int_{0}^{\infty}\left[v_{0}(x)-V(x+\alpha-\beta)\right] d x=-\int_{0}^{\infty} U(-s \tau+\alpha-\beta) d \tau .
$$

On the other hand, by applying a similar argument to the second equation of (1.1) in the form

$$
(u-U)_{t}=-\left(p(v)-p(V)-\mu \frac{u_{x}}{v}+\mu \frac{U^{\prime}}{V}\right)_{x},
$$

we find that

$$
\begin{gathered}
\int_{0}^{\infty}\left[u_{0}(x)-U(x+\alpha-\beta)\right] d x+\int_{0}^{\infty}[p(v(0, t))-p(V(-s t+\alpha-\beta))] d t \\
-\mu \int_{0}^{\infty}\left[\frac{u_{x}(0, t)}{v(0, t)}-\frac{U^{\prime}(-s t+\alpha-\beta)}{V(-s t+\alpha-\beta)}\right] d t=0 .
\end{gathered}
$$


However, as stated in the introduction, we expect that $v(0, t)$ and $u_{x}(0, t)=v_{t}(0, t)$ are automatically controlled by the effects of boundary and viscosity so that (2.14) holds with the same shift $\alpha$ defined by (2.11). This situation is really possible because $v(0, t)$ is not specified.

It is interesting to compare our case with the scalar case

$$
u_{t}+f(u)_{x}=u_{x x},\left.\quad u\right|_{x=0}=u_{-},\left.\quad u\right|_{x=\infty}=u_{+} \quad\left(u_{-}>u_{+}\right),
$$

which is considered by LIU \& YU [9] for $f(u)=\frac{1}{2} u^{2}$ and by LIU \& NISHIHARA [8] for the general flux function $f(u)$. To locate the shift $\alpha$ they had to control the value $\int_{0}^{\infty} u_{x}(0, t) d t$, as suggested by the form (2.14). They chose the shift $\alpha$ as a function depending on time $t$, say $d(t)$, and studied it by getting pointwise estimates via the Green function in [9] or by getting technical weighted energy estimates in [8], where the decay rate estimates on $d(t)$ also played important role. But the same argument cannot be applied straightforwardly to the case of systems, even for the simpler $2 \times 2$ system, e.g., our $p$-system with vsicosity, because the shift function $d(t)$ is overdetermined by two equations, a situation that is even worse for $n \times n$ conservation laws. However, in view of the observations 1 and 2 stated in the Introduction, we can reasonably conjecture that the amount of shift $\alpha$ is a constant for our problem, just as we have shown in (2.11). This is one of the key points in this paper.

\subsection{Main Result}

To state the main theorem precisely, we suppose that for some $\beta>0$,

$$
v_{0}(x)-V(x-\beta) \in H^{1} \cap L^{1}, \quad u_{0}(x)-U(x-\beta) \in H^{1} \cap L^{1}
$$

and suppose that the compatibility condition

holds. Setting

$$
u_{0}(0)=0
$$

$$
\left(\Phi_{0}, \Psi_{0}\right)(x)=-\int_{x}^{\infty}\left(v_{0}(y)-V(y-\beta), u_{0}(y)-U(y-\beta)\right) d y,
$$

we further assume that

$$
\left(\Phi_{0}, \Psi_{0}\right) \in L^{2} .
$$

We note an asymptotic property of the constant shift $\alpha$, before we state the main theorem.

Lemma 2.2. Under the assumptions (2.15), (2.16) and (2.18), $\left(\Phi_{0}, \Psi_{0}\right) \in H^{2}$ and the shift $\alpha$ defined by (2.11) satisfies $\alpha \rightarrow 0$ as $\left\|\left(\Phi_{0}, \Psi_{0}\right)\right\|_{2} \rightarrow 0$ and $\beta \rightarrow+\infty$.

Proof. It is easy to see that $\left(\Phi_{0}, \Psi_{0}\right) \in H^{2}$ from (2.15) and (2.18). Since $0<$ $-U(-s t-\beta) \leqq C e^{-c_{-}(s t+\beta)}$ (see (2.4), (2.5)) and since $\beta>0$, so that $\left|\int_{0}^{\infty} U(-s t-\beta) d t\right| \leqq C e^{-c_{-} \beta}$, we obtain from formula (2.11) for the shift $\alpha$ that

$$
|\alpha| \leqq C\left(\left|\Phi_{0}(0)\right|+e^{-c_{-} \beta}\right) \leqq C\left(\left\|\Phi_{0}\right\|_{2}+e^{-c_{-} \beta}\right) \rightarrow 0
$$

as $\beta \rightarrow+\infty$ and $\left\|\left(\Phi_{0}, \Psi_{0}\right)\right\|_{2} \rightarrow 0$. 
Now, we state our main theorem.

Theorem 2.3. For any $u_{+}<0$ and $v_{+}>0$, suppose that assumptions (2.15), (2.16), and (2.18) hold. Furthermore, let

$$
(\gamma-1)^{2}\left(v_{+}-v_{-}\right)<2 \gamma v_{-},
$$

where $v_{-}\left(v_{+}>v_{-}>0\right)$ and $s>0$ are defined by (1.6). Then there exists a positive constant $\varepsilon_{0}$ such that if $\left\|\left(\Phi_{0}, \Psi_{0}\right)\right\|_{2}+\beta^{-1}<\varepsilon_{0}$, then (1.1)-(1.3) has a unique global solution $(v, u)(x, t)$ satisfying

$$
\begin{aligned}
& v(x, t)-V(x-s t+\alpha-\beta) \in C^{0}\left([0, \infty) ; H^{1}\right) \cap L^{2}\left(0, \infty ; H^{1}\right), \\
& u(x, t)-U(x-s t+\alpha-\beta) \in C^{0}\left([0, \infty) ; H^{1}\right) \cap L^{2}\left(0, \infty ; H^{2}\right)
\end{aligned}
$$

and the asymptotic behavior

$$
\sup _{x \in R_{+}}|(v, u)(x, t)-(V, U)(x-s t+\alpha-\beta)| \rightarrow 0 \quad \text { as } t \rightarrow \infty,
$$

where $\alpha=\alpha(\beta)$ is determined by (2.11).

Remark. Condition (2.19) is much weaker than those in MATSUMURA \& NishiHARA [12] and LIU \& WANG [10], even for the Cauchy problem. In fact, both of their conditions in $[12,10]$ imply that $v_{+}-v_{-}<C(\gamma-1)^{-1}$, but our condition is $v_{+}-v_{-}<C \gamma(\gamma-1)^{-2}$. We are indebted to Professor SHUICHI KaWASHIMA for his helpful suggestion [3] for this weaker condition.

\section{Reformulation of the Original Problem}

Following the argument in Subsection 2.2, let us continue a heuristic argument for the solution. Define new unknown functions $\phi(x, t)$ and $\psi(x, t)$ by

$$
\left.\begin{array}{l}
\phi(x, t)=-\int_{x}^{\infty}[v(y, t)-V(y-s t+\alpha-\beta)] d y \\
\psi(x, t)=-\int_{x}^{\infty}[u(y, t)-U(y-s t+\alpha-\beta)] d y
\end{array}\right\} \quad(x, t) \in R_{+} \times R_{+},
$$

which means that we look for the solution $(v, u)(x, t)$ in the form

$$
\begin{aligned}
& v(x, t)=\phi_{x}(x, t)+V(x-s t+\alpha-\beta), \\
& u(x, t)=\psi_{x}(x, t)+U(x-s t+\alpha-\beta) .
\end{aligned}
$$

Substituting (3.2) into (1.1), and integrating the system on $[x, \infty)$ with respect to $x$, we obtain the system for $(\phi, \psi)(x, t)$ in the form

$$
\begin{aligned}
\phi_{t}-\psi_{x} & =0 \\
\psi_{t}+p\left(V+\phi_{x}\right)-p(V) & =\mu\left(\frac{U^{\prime}+\psi_{x x}}{V+\phi_{x}}-\frac{U^{\prime}}{V}\right) .
\end{aligned}
$$


By (3.1), the initial data satisfy

$$
\begin{aligned}
\phi(x, 0) & =-\int_{x}^{\infty}\left[v_{0}(y)-V(y+\alpha-\beta)\right] d y \\
& =\Phi_{0}(x)+\int_{x}^{\infty}[V(y+\alpha-\beta)-V(y-\beta)] d y \\
& =\Phi_{0}(x)+\int_{x}^{\infty} \int_{0}^{\alpha} V^{\prime}(y+\theta-\beta) d \theta d y \\
& =\Phi_{0}(x)+\int_{0}^{\alpha}\left[v_{+}-V(x+\theta-\beta)\right] d \theta \\
& =: \phi_{0}(x), \\
\psi(x, 0) & =-\int_{x}^{\infty}\left[u_{0}(y)-U(y+\alpha-\beta)\right] d y \\
& =\Psi_{0}(x)+\int_{x}^{\infty}[U(y+\alpha-\beta)-U(y-\beta)] d y \\
& =\Psi_{0}(x)+\int_{0}^{\alpha}\left[u_{+}-U(x+\theta-\beta)\right] d \theta \\
& =: \psi_{0}(x) .
\end{aligned}
$$

The initial perturbations (3.4) and (3.5) satisfy

Lemma 3.1. Under the assumptions (2.15), (2.16) and (2.18), the initial perturbation $\left(\phi_{0}, \psi_{0}\right) \in H^{2}$ and satisfies

$$
\left\|\left(\phi_{0}, \psi_{0}\right)\right\|_{2} \rightarrow 0 \quad \text { as }\left\|\left(\Phi_{0}, \Psi\right)\right\|_{2} \rightarrow 0 \text { and } \beta \rightarrow+\infty .
$$

Proof. Let $\chi_{1}(x):=\int_{0}^{\alpha}\left[v_{+}-V(x-\beta+\theta)\right] d \theta$. Then it follows from (2.5) that

$$
\left|v_{+}-V(x-\beta+\theta)\right| \leqq C e^{-c_{+}|x-\beta+\theta|} \leqq C e^{-c_{+}|x-\beta|} e^{c_{+}|\alpha|} \leqq C e^{-c_{+}|x-\beta|}
$$

for $|\alpha|<1$ (see Lemma 2.2). Hence, we have

$$
\begin{aligned}
\left\|\chi_{1}\right\|^{2} & \leqq C \int_{0}^{\infty} \alpha^{2} e^{-2 c_{+}|x-\beta|} d x \\
& =C \alpha^{2}\left[\int_{0}^{\beta} e^{-2 c_{+}(\beta-x)} d x+\int_{\beta}^{\infty} e^{-2 c_{+}(x-\beta)} d x\right] \\
& =\frac{C \alpha^{2}}{2 c_{+}}\left[2-e^{-2 c_{+} \beta}\right] \leqq C \alpha^{2} / c_{+}
\end{aligned}
$$

where $C$ is independent of $\alpha$ and $\beta$. Similarly, we can prove that $\left\|\chi_{1}^{\prime}\right\|^{2} \leqq C \alpha^{2}$ and $\left\|\chi_{1}^{\prime \prime}\right\|^{2} \leqq C \alpha^{2}$. Thus, we proved $\left\|\chi_{1}\right\|_{2} \leqq C|\alpha|$. In the same way, we have that 
$\chi_{2}(x):=\int_{0}^{\alpha}\left[u_{+}-U(x+\theta-\beta)\right] d \theta$ satisfies $\left\|\chi_{2}\right\|_{2} \leqq C|\alpha|$. Thus, using Lemma 2.2 , we have

$\left\|\left(\phi_{0}, \psi_{0}\right)\right\|_{2} \leqq\left\|\left(\Phi_{0}, \Psi_{0}\right)\right\|_{2}+\left\|\left(\chi_{1}, \chi_{2}\right)\right\|_{2} \leqq C\left(\left\|\left(\Phi_{0}, \Psi_{0}\right)\right\|_{2}+|\alpha|\right)$,

which tends to zero as $\beta \rightarrow+\infty$ and $\left\|\left(\Phi_{0}, \Psi_{0}\right)\right\|_{2} \rightarrow 0$.

By (3.1), (3.2) and (2.12) the boundary data satisfy

$$
\begin{aligned}
\phi(0, t) & =\int_{t}^{\infty} U(-s \tau+\alpha-\beta) d \tau \equiv: A(t), \\
\phi_{0}(0) & =\int_{0}^{\infty} U(-s \tau+\alpha-\beta) d \tau=A(0), \\
\psi_{x}(0, t) & =u(0, t)-U(-s t+\alpha-\beta)=-U(-s t+\alpha-\beta)=A^{\prime}(t) .
\end{aligned}
$$

Note that if (3.8) and (3.9) hold, then (3.7) automatically holds by the equation $\phi_{t}-\psi_{x}=0$. Hence, we regard (3.9) as a Neumann boundary condition for $\psi$ and (3.8) as a restriction on the initial data $\phi_{0}$. Under these considerations, we rewrite the system (3.3) in the form

$$
\left.\begin{array}{rl}
\phi_{t}-\psi_{x} & =0 \\
\psi_{t}-f(V) \phi_{x}-\frac{\mu}{V} \psi_{x x} & =F
\end{array}\right\} \quad(x, t) \in R_{+} \times R_{+},
$$

with the initial conditions (3.4) and (3.5) and Neumann boundary condition (3.9) as

$$
\begin{gathered}
\left.(\phi, \psi)\right|_{t=0}=\left(\phi_{0}, \psi_{0}\right)(x) \in H^{2}, \quad x \geqq 0, \\
\left.\psi_{x}\right|_{x=0}=A^{\prime}(t), \quad t \geqq 0, \\
\phi_{0}(0)=A(0),
\end{gathered}
$$

where $A(t)=\int_{t}^{\infty} U(-s \tau+\alpha-\beta) d \tau$ and

$$
\begin{aligned}
f(V)= & -p^{\prime}(V)+\frac{\mu s V_{x}}{V^{2}}=\frac{h(V)-p^{\prime}(V) V}{V} \equiv \frac{K(V)}{V}, \\
F= & -\left\{p\left(V+\phi_{x}\right)-p(V)-p^{\prime}(V) \phi_{x}\right\} \\
& -\left(\mu \psi_{x x}+h(V) \phi_{x}\right)\left(\frac{1}{V+\phi_{x}}-\frac{1}{V}\right) .
\end{aligned}
$$

Conversely, once we prove that the initial-boundary-value problem (3.10) and (3.11) has a unique global solution $(\phi, \psi)(x, t)$ in $C\left([0,+\infty) ; H^{2}\right)$, then we can have a unique global solution $(v, u)(x, t)$ of the original initial-boundary-value problem (1.1)-(1.3) in $C\left([0,+\infty) ; H^{1}\right)$ by (3.2). 
For any interval $I \subset R_{+}$, we define the solution space $X(I)$ by

$$
\begin{aligned}
X(I)= & \left\{(\phi, \psi) \in C^{0}\left(I ; H^{2}\right) ; \phi_{x} \in L^{2}\left(I ; H^{1}\right),\right. \\
& \left.\psi_{x} \in L^{2}\left(I ; H^{2}\right), \quad \sup _{t \in I}\|(\phi, \psi)(t)\|_{2} \leqq \delta_{0}\right\},
\end{aligned}
$$

where $\delta_{0}=\frac{1}{2} v_{-}$, and set

$$
N(t)=\sup _{0 \leqq \tau \leqq t}\left(\|\phi(\tau)\|_{2}+\|\psi(\tau)\|_{2}\right), \quad N_{0}=\left\|\phi_{0}\right\|_{2}+\left\|\psi_{0}\right\|_{2} .
$$

By the Sobolev lemma,

$$
\sup _{x}|f(x)| \leqq\|f\|_{1} \quad \text { for } \quad f \in H^{1} .
$$

Note that if $(\phi, \psi) \in X([0, T])$ for $T \in R_{+}$, then

$$
\left(V+\phi_{x}\right)(x, t) \geqq v_{-}-\left\|\phi_{x}\right\|_{1} \geqq \frac{1}{2} v_{-}, \quad(x, t) \in R_{+} \times[0, T],
$$

which ensures that the system (3.10) is uniformly nonsingular on $[0, T]$, and

$$
|F|=O\left(\left|\phi_{x}\right|^{2}+\left|\phi_{x}\right|\left|\psi_{x x}\right|\right) \text {. }
$$

Then, corresponding to Theorem 2.3, we give the following theorem for the initialboundary-value problem (3.10) and (3.11).

Theorem 3.2. Suppose that the assumptions of Theorem 2.3 hold. Then there exists a positive constant $\varepsilon_{1}$ such that if $N_{0}+\beta^{-1} \leqq \varepsilon_{1}$, then the initial-boundaryvalue problem (3.10) and (3.11) has a unique global solution $(\phi, \psi) \in X([0, \infty))$ satisfying

$$
\begin{gathered}
\|(\phi, \psi)(t)\|_{2}^{2}+\int_{0}^{t}\left\{\left\|\phi_{x}(\tau)\right\|_{1}^{2}+\left\|\psi_{x}(\tau)\right\|_{2}^{2}\right\} d \tau \\
\leqq C\left(\left\|\left(\phi_{0}, \psi_{0}\right)\right\|_{2}^{2}+e^{-c_{-} \beta}\right), \\
\int_{0}^{t}\left|\frac{d}{d t}\left\|\phi_{x}(\tau)\right\|^{2}\right|+\left|\frac{d}{d t}\left\|\psi_{x}(\tau)\right\|^{2}\right| d \tau \leqq C\left(\left\|\left(\phi_{0}, \psi_{0}\right)\right\|_{2}^{2}+e^{-c_{-} \beta}\right),
\end{gathered}
$$

for any $t \geqq 0$. Moreover, the solution is asymptotically stable:

$$
\sup _{x \in R_{+}}\left|\left(\phi_{x}, \psi_{x}\right)(x, t)\right| \rightarrow 0 \quad \text { as } \quad t \rightarrow \infty
$$

Theorem 2.3 easily follows from Theorem 3.2. Therefore, our purpose is now to prove Theorem 3.2. We now state the local existence result and the a priori estimates for the initial-boundary-value problem (3.10) and (3.11) as follows.

Proposition 3.3 (Local Existence). For any $\tau \geqq 0$, consider the problem

$$
\left.\begin{array}{r}
\phi_{t}-\psi_{x}=0 \\
\psi_{t}-f(V) \phi_{x}-\frac{\mu}{V} \psi_{x x}=F
\end{array}\right\} \quad x \in R_{+}, t \geqq \tau,
$$


with initial and boundary conditions

$$
\begin{gathered}
(\phi, \psi)(x, \tau)=\left(\phi_{\tau}, \psi_{\tau}\right)(x) \in H^{2}, \\
\psi_{x}(0, t)=A^{\prime}(t), \quad t \geqq \tau,
\end{gathered}
$$

subject to the compatibility condition $\psi_{\tau, x}(0, \tau)=A^{\prime}(\tau)$. Then there exists a positive constant $C_{0}$ independent of $\tau$ such that: For any $\delta \in\left(0, \delta_{0} / C_{0}\right]$ and $\beta>1$, there exists a positive constant $T_{0}$ depending on $\delta$ and $\beta$ but not on $\tau$ such that, if $\left\|\left(\phi_{\tau}, \psi_{\tau}\right)\right\| \leqq \delta$ and $\sup _{t \geq 0}\left(\left|A^{\prime}(t)\right|+\left|A^{\prime \prime}(t)\right|\right) \leqq \delta$, then the problem (3.18) and (3.19) has a unique solution $(\phi, \psi) \in X\left(\left[\tau, \tau+T_{0}\right]\right)$ satisfying $\|(\phi, \psi)(t)\|_{2} \leqq$ $C_{0} \delta$ for $t \in\left[\tau, \tau+T_{0}\right]$.

Proposition 3.4 (A Priori Estimates). Let $(\phi, \psi) \in X([0, T])$ be a solution of (3.10) and (3.11) for a positive T. Then there exist positive constants $\delta_{1}\left(\leqq \delta_{0}\right)$ and $C_{1}$, which are independent of $T$, such that if $N(T)<\delta_{1}$, then $(\phi, \psi)(x, t)$ satisfies the a priori estimates (3.15) and (3.16) with $C=C_{1}$ for $0 \leqq t \leqq T$.

We omit the proof of Proposition 3.3 because it can be shown in a standard way. The proof of Proposition 3.4 is a key for Theorem 3.2; it will be obtained in the next section.

Proof of Theorem 3.2. Based on the repeated use of Propositions 3.3 and 3.4, the standard continuation argument asserts the existence of a unique global solution $(\phi, \psi) \in X([0, \infty))$ satisfying (3.15) and (3.16) for any $t \in[0, \infty)$, provided that $\left\|\left(\phi_{0}, \psi_{0}\right)\right\|_{2}$ and $\beta^{-1}$ are chosen so small that

$$
\begin{aligned}
\left\|\left(\phi_{0}, \psi_{0}\right)\right\|_{2} \leqq & \delta_{1} / C_{0}, \quad C_{1}\left(\left\|\left(\phi_{0}, \psi_{0}\right)\right\|_{2}^{2}+e^{-c_{-} \beta}\right) \leqq\left(\delta_{1} / C_{0}\right)^{2}, \\
& \sup _{t \geqq 0}\left(\left|A^{\prime}(t)\right|+\left|A^{\prime \prime}(t)\right|\right) \leqq \delta_{1} / C_{0} .
\end{aligned}
$$

To prove (3.17), we consider the function $\mathbf{H}(t):=\left\|\left(\phi_{x}, \psi_{x}\right)(t)\right\|^{2}$. By virtue of the uniform estimates (3.15) and (3.16), we see that both $\mathbf{H}(t)$ and $\left|\mathbf{H}^{\prime}(t)\right|$ are integrable over $t \geqq 0$. Thus $\mathbf{H}(t) \rightarrow 0$, i.e., $\left\|\left(\phi_{x}, \psi_{x}\right)(t)\right\| \rightarrow 0$, as $t \rightarrow \infty$. Furthermore, $\left\|\left(\phi_{x x}, \bar{\psi}_{x x}\right)(t)\right\|$ is uniformly bounded for $t \geqq 0$ due to (3.15). By the Sobolev inequality, we then obtain

$$
\sup _{x \in R_{+}}\left|\left(\phi_{x}, \psi_{x}\right)(x, t)\right|^{2} \leqq 2\left\{\left\|\phi_{x}(t)\right\|\left\|\phi_{x x}(t)\right\|+\left\|\psi_{x}(t)\right\|\left\|\psi_{x x}(t)\right\|\right\} \rightarrow 0
$$

as $t \rightarrow \infty$. This completes the proof of Theorem 3.2.

\section{Proof of the a Priori Estimates}

Let $(\phi, \psi) \in X([0, T])$ be a solution of (3.10) and (3.11) for a positive constant $T$. Without loss of generality, we may restrict $N(T)<\delta_{0}, \beta>1$ and $|\alpha|<1$. Throughout this section, we use the letter $C$ to denote some positive constant which is independent of $T, \beta$ and $\alpha$. We first give the boundary estimates. 
Lemma 4.1. For $0 \leqq t \leqq T$, the following inequalities hold:

$$
\begin{gathered}
\left.\left|\int_{0}^{t}(\phi \psi)\right|_{x=0} d \tau\left|\leqq C e^{-c_{-} \beta}, \quad\right| \int_{0}^{t}\left(\psi \psi_{x}\right)\right|_{x=0} d \tau \mid \leqq C e^{-c_{-} \beta} \\
\left.\left|\int_{0}^{t}\left(\phi_{x} \psi_{x}\right)\right|_{x=0} d \tau\left|\leqq C e^{-c_{-} \beta}, \quad\right| \int_{0}^{t}\left(\psi_{x} \psi_{t}\right)\right|_{x=0} d \tau \mid \leqq C e^{-c_{-} \beta} \\
\left.\left|\int_{0}^{t}\left(\psi_{x} \psi_{x x}\right)\right|_{x=0} d \tau\left|\leqq C e^{-c_{-} \beta}, \quad\right| \int_{0}^{t}\left(\psi_{x t} \psi_{x x}\right)\right|_{x=0} d \tau \mid \leqq C e^{-c_{-} \beta}
\end{gathered}
$$

where $c_{-}=\left|p^{\prime}\left(v_{-}\right)+s^{2}\right| v_{-} / \mu s>0$ is as in (2.5).

Proof. We need some preliminary results: From the first equation of (3.10) and the Neumann boundary condition in (3.11), we have $\left.\phi_{t}\right|_{x=0}=\left.\psi_{x}\right|_{x=0}=-U(-s t+$ $\alpha-\beta)$. We integrate this equation with respect to $t$ to get

$$
\begin{aligned}
\phi(0, t) & =\phi_{0}(0)+\int_{0}^{t} A^{\prime}(\tau) d \tau \\
& =\int_{t}^{\infty} U(-s \tau+\alpha-\beta) d \tau=A(t) .
\end{aligned}
$$

Since $|-s t+\alpha-\beta|=s t+\beta-\alpha$ because $s>0$ and $\beta-\alpha>0(\beta>1,|\alpha|<1)$, and since $|U(-s t+\alpha-\beta)| \leqq C e^{-c_{-}|-s t+\alpha-\beta|}=C e^{-c_{-}(\beta-\alpha)} e^{-c_{-} s t} \leqq C e^{-c_{-} \beta} e^{-c_{-} s t}$ (see (2.5)), we have

$$
|\phi(0, t)|=|A(t)| \leqq O(1) e^{-c_{-} \beta} e^{-c_{-} s t} .
$$

Since $\left.\psi_{x}\right|_{x=0}=-U(-s t+\alpha-\beta)=A^{\prime}(t)$ from the definitions (3.9) and (4.4), we have $\left.\psi_{x t}\right|_{x=0}=A^{\prime \prime}(t)$. Similarly, using (2.2) (or (2.3)), (2.5), and $|-s t+\alpha-\beta|=$ $s t+\beta-\alpha$, we can conclude that $A(t) \in W^{3,1}(0, \infty)$ and

$$
\begin{aligned}
\left|\frac{d^{k}}{d t^{k}} A(t)\right| & \leqq C e^{-c_{-} \beta} e^{-c_{-} s t}, \quad k=0,1,2,3, \\
\|A\|_{W^{3,1}} & \leqq C e^{-c_{-} \beta} .
\end{aligned}
$$

The following estimates obtained by the Sobolev lemma are also used in what follows:

$$
\begin{aligned}
&|\psi(0, t)| \leqq \sup _{x \in R_{+}}|\psi(x, t)| \leqq C N(T) \leqq C, \\
&\left|\phi_{x}(0, t)\right| \leqq \sup _{x \in R_{+}}\left|\phi_{x}(x, t)\right| \leqq C N(T) \leqq C,
\end{aligned}
$$

where we have assumed that $N(T)<\delta_{0}$.

Let us give the proofs of (4.1)-(4.3). Using (4.4)-(4.7), we have the first inequality of (4.1) for the boundary value:

$$
\begin{aligned}
\left|\int_{0}^{t}(\phi \psi)\right|_{x=0} d \tau \mid & \leqq \int_{0}^{t}|A(\tau)||\psi(0, \tau)| d \tau \\
& \leqq C N(T) \int_{0}^{t}|A(\tau)| d \tau \leqq C e^{-c_{-} \beta}
\end{aligned}
$$


14

A. Matsumura \& Ming Me

A similar computation yields the second inequality of (4.1) and the first inequality of (4.2):

$$
\begin{aligned}
\left|\int_{0}^{t}\left(\psi \psi_{x}\right)\right|_{x=0} d \tau \mid & \leqq \int_{0}^{t}\left|A^{\prime}(\tau)\right||\psi(0, \tau)| d \tau \\
& \leqq C N(T) \int_{0}^{t}\left|A^{\prime}(\tau)\right| d \tau \leqq C e^{-c_{-} \beta} \\
\left|\int_{0}^{t}\left(\phi_{x} \psi_{x}\right)\right|_{x=0} d \tau \mid & \leqq \int_{0}^{t}\left|A^{\prime}(\tau)\right|\left|\phi_{x}(0, \tau)\right| d \tau \\
& \leqq C N(T) \int_{0}^{t}\left|A^{\prime}(\tau)\right| d \tau \leqq C e^{-c_{-} \beta}
\end{aligned}
$$

To prove the other boundary estimates, we make use of $\phi_{t x}=\psi_{x x}$, integration by parts, and (4.6), (4.7), to obtain

$$
\begin{aligned}
\left|\int_{0}^{t}\left(\psi_{x} \psi_{t}\right)\right|_{x=0} d \tau \mid & =\left|\int_{0}^{t} A^{\prime}(\tau) \psi_{t}(0, \tau) d \tau\right| \\
& =\left|\int_{0}^{t}\left[\left\{A^{\prime}(\tau) \psi(0, \tau)\right\}_{t}-A^{\prime \prime}(\tau) \psi(0, \tau)\right] d \tau\right| \\
& \leqq\left|A^{\prime}(t) \psi(0, t)\right|+\left|A^{\prime}(0) \psi_{0}(0)\right|+\int_{0}^{t}\left|A^{\prime \prime}(\tau)\right||\psi(0, \tau)| d \tau \\
& \leqq C N(T)\left[\left|A^{\prime}(t)\right|+\left|A^{\prime}(0)\right|+\int_{0}^{t}\left|A^{\prime \prime}(\tau)\right| d \tau\right] \\
& \leqq C e^{-c_{-} \beta}
\end{aligned}
$$

$$
\begin{aligned}
\left|\int_{0}^{t}\left(\psi_{x} \psi_{x x}\right)\right|_{x=0} d \tau \mid= & \left|\int_{0}^{t} A^{\prime}(\tau) \psi_{x x}(0, \tau) d \tau\right|=\left|\int_{0}^{t} A^{\prime}(\tau) \phi_{x t}(0, \tau) d \tau\right| \\
= & \left|\int_{0}^{t}\left[\left\{A^{\prime}(\tau) \phi_{x}(0, \tau)\right\}_{t}-A^{\prime \prime}(\tau) \phi_{x}(0, \tau)\right] d \tau\right| \\
\leqq & \left|A^{\prime}(t)\right|\left|\phi_{x}(0, t)\right|+\left|A^{\prime}(0)\right|\left|\phi_{x}(0,0)\right| \\
& +\int_{0}^{t}\left|A^{\prime \prime}(\tau)\right|\left|\phi_{x}(0, \tau)\right| d \tau \\
\leqq & C N(T)\left[\left|A^{\prime}(t)\right|+\left|A^{\prime}(0)\right|+\int_{0}^{t}\left|A^{\prime \prime}(\tau)\right| d \tau\right] \\
\leqq & C e^{-c_{-} \beta}
\end{aligned}
$$




$$
\begin{aligned}
\left|\int_{0}^{t}\left(\psi_{x t} \psi_{x x}\right)\right|_{x=0} d \tau \mid= & \left|\int_{0}^{t} A^{\prime \prime}(\tau) \psi_{x x}(0, \tau) d \tau\right|=\left|\int_{0}^{t} A^{\prime \prime}(\tau) \phi_{x t}(0, \tau) d \tau\right| \\
= & \left|\int_{0}^{t}\left[\left\{A^{\prime \prime}(\tau) \phi_{x}(0, \tau)\right\}_{t}-A^{\prime \prime \prime}(\tau) \phi_{x}(0, \tau)\right] \tau\right| \\
\leqq & \left|A^{\prime \prime}(t)\right|\left|\phi_{x}(0, t)\right|+\left|A^{\prime \prime}(0)\right|\left|\phi_{x}(0,0)\right| \\
& +\int_{0}^{t}\left|A^{\prime \prime \prime}(\tau)\right|\left|\phi_{x}(0, \tau)\right| d \tau \\
\leqq & C N(T)\left[\left|A^{\prime \prime}(t)\right|+\left|A^{\prime \prime}(0)\right|+\int_{0}^{t}\left|A^{\prime \prime \prime}(\tau)\right| d \tau\right] \\
\leqq & C e^{-c_{-} \beta} .
\end{aligned}
$$

We now establish the a priori estimates. We first obtain

Lemma 4.2. Suppose $V(x-s t+\alpha-\beta)$ is the viscous shock profile. Then

$$
\begin{gathered}
0 \leqq \frac{h(V)}{V} \leqq \frac{s^{2}\left(v_{+}-v_{-}\right)}{v_{-}} \\
0<-p^{\prime}\left(v_{+}\right) \leqq f(V) \leqq-p^{\prime}\left(v_{-}\right)+\frac{s^{2}\left(v_{+}-v_{-}\right)}{v_{-}} \equiv: c_{0} \\
f(V)-\frac{h(V)}{2 V} \geqq-p^{\prime}\left(v_{+}\right)>0 .
\end{gathered}
$$

Proof. Since $0<v_{-}<V<v_{+}$and $p(V)=a V^{-\gamma}$, we easily find that

$$
p\left(v_{-}\right)>p(V)>p\left(v_{+}\right)>0, \quad-p^{\prime}\left(v_{-}\right)>-p^{\prime}(V)>-p^{\prime}\left(v_{+}\right)>0 .
$$

Thus, $h(V)=p\left(v_{-}\right)-s^{2}\left(V-v_{-}\right)-p(V) \leqq p\left(v_{-}\right)-p\left(v_{+}\right)=s^{2}\left(v_{+}-v_{-}\right)$ and $\frac{h(V)}{V} \leqq \frac{s^{2}\left(v_{+}-v_{-}\right)}{v_{-}}$. This proves (4.8). By the definition of $f(V)$ and by (4.8) and (4.11), we get (4.9). Furthermore,

$$
f(V)-\frac{h(V)}{2 V}=-p^{\prime}(V)+\frac{h(V)}{2 V} \geqq-p^{\prime}\left(v_{+}\right),
$$

due to (4.8) and (4.11). This proves (4.10).

Next, we have the following basic energy estimate.

Lemma 4.3. For $t \in[0, T]$,

$$
\begin{aligned}
& \|(\phi, \psi)(t)\|^{2}+\int_{0}^{t}\left\|\psi_{x}(\tau)\right\|^{2} d \tau \\
& \quad \leqq C\left\{\left\|\left(\phi_{0}, \psi_{0}\right)\right\|^{2}+e^{-c_{-} \beta}+N(T) \int_{0}^{t}\left[\left\|\phi_{x}(\tau)\right\|^{2}+\left\|\psi_{x x}(\tau)\right\|^{2}\right] d \tau\right\} .
\end{aligned}
$$


Proof. Multiplying the first equation of (3.10) by $\phi$ and the second of (3.10) by $f(V)^{-1} \psi$ (where $f(V)=K(V) / V$; see (3.12)), and adding these equalities, we obtain

$$
\begin{gathered}
\left\{\frac{1}{2} \phi^{2}\right\}_{t}-\{\phi \psi\}_{x}+\left\{\frac{1}{2 f(V)} \psi^{2}\right\}_{t}+\frac{s}{2}\left(\frac{V}{K(V)}\right)^{\prime} V_{x} \psi^{2}-\left\{\frac{\mu}{K(V)} \psi \psi_{x}\right\}_{x} \\
+\frac{\mu}{K(V)} \psi_{x}^{2}-\frac{\mu K^{\prime}(V) V_{x}}{K(V)^{2}} \psi \psi_{x}=F \cdot \frac{V \psi}{K(V)} .
\end{gathered}
$$

Since

$$
\left|\frac{\mu K^{\prime}(V) V_{x}}{K(V)^{2}} \psi \psi_{x}\right| \leqq \varepsilon \frac{\mu}{K(V)} \psi_{x}^{2}+\frac{\mu K^{\prime}(V)^{2} V_{x}^{2}}{4 \varepsilon K(V)^{3}} \psi^{2}
$$

for any $\varepsilon>0$, which will be determined later, substituting this inequality into (4.13) yields

$$
\begin{aligned}
&\left\{\frac{1}{2} \phi^{2}\right.\left.+\frac{1}{2 f(V)} \psi^{2}\right\}_{t}-\left\{\phi \psi+\frac{\mu}{K(V)} \psi \psi_{x}\right\}_{x} \\
&+(1-\varepsilon) \frac{\mu}{K(V)} \psi_{x}^{2}+Z(V) V_{x} \psi^{2} \leqq F \cdot \frac{V \psi}{K(V)},
\end{aligned}
$$

where

$$
Z(V)=\frac{s}{2}\left(\frac{V}{K(V)}\right)^{\prime}-\frac{\mu K^{\prime}(V)^{2} V_{x}}{4 \varepsilon K(V)^{3}} .
$$

In view of (2.3), (3.12) and $p(V)=a V^{-\gamma}$, a tedious but straightforward computation gives

$$
\begin{aligned}
Z(V)= & \frac{1}{4 s K(V)^{3}}\left\{2 s^{2}\left[\gamma^{3} p(V)^{2}+h(V)^{2}+\gamma s^{2} V p(V)\right]\right. \\
& +2 s^{2}\left[\gamma(\gamma+1)-(2 \varepsilon)^{-1} \gamma(\gamma-1)\right] p(V) h(V) \\
& \left.-\frac{\gamma^{2}(\gamma-1)^{2} h(V)}{\varepsilon V} p(V)^{2}+2 s^{4}\left[1-(2 \varepsilon)^{-1}\right] V h(V)\right\} .
\end{aligned}
$$

Substituting (4.8) into (4.16) yields

$$
\begin{aligned}
Z(V) \geqq & \frac{1}{4 s K(V)^{3}}\left\{2 s^{2}\left[h(V)^{2}+\gamma s^{2} V p(V)\right]\right. \\
& +2 s^{2} \gamma^{2}\left[\gamma-\frac{(\gamma-1)^{2}\left(v_{+}-v_{-}\right)}{\varepsilon v_{-}}\right] p(V)^{2} \\
& +2 s^{2} \gamma\left[(\gamma+1)-(2 \varepsilon)^{-1}(\gamma-1)\right] p(V) h(V) \\
& \left.+2 s^{4}\left[1-(2 \varepsilon)^{-1}\right] V h(V)\right\} .
\end{aligned}
$$


Using the sufficient condition (2.19), and choosing $\varepsilon$ as

$$
\max \left\{\frac{(\gamma-1)^{2}\left(v_{+}-v_{-}\right)}{2 \gamma v_{-}}, \frac{1}{2}\right\} \leqq \varepsilon<1,
$$

we have

$$
Z(V) \geqq C>0 .
$$

Integrating (4.14) over $[0, \infty) \times[0, t]$, we have

$$
\begin{aligned}
& \int_{0}^{\infty}\left[\phi^{2}+f(V)^{-1} \psi^{2}\right] d x+2(1-\varepsilon) \mu \int_{0}^{t} \int_{0}^{\infty} K(V)^{-1} \psi_{x}^{2} d x d \tau \\
& \quad+2 \int_{0}^{t} \int_{0}^{\infty} Z(V) V_{x} \psi^{2} d x d \tau \\
& \leqq \int_{0}^{\infty}\left[\phi_{0}^{2}+f(V(x+\alpha-\beta))^{-1} \psi_{0}^{2}\right] d x \\
& \quad+2\left|\int_{0}^{t}\left(\phi \psi+\frac{\mu}{K(V)} \psi \psi_{x}\right)\right|_{x=0} d \tau\left|+2 \int_{0}^{t} \int_{0}^{\infty}\right| F V K(V)^{-1} \psi \mid d x d \tau .
\end{aligned}
$$

Using (4.18), $V_{x}>0(\operatorname{see}(2.4)), v_{-}\left|p^{\prime}\left(v_{+}\right)\right| \leqq K(V) \leqq c_{0} v_{+}, f(V)^{-1} \geqq c_{0}^{-1}$ (see (4.9)), and the boundary estimates (4.1) and (4.3), we get the basic energy estimate (4.12).

Lemma 4.4. For $t \in[0, T]$,

$$
\begin{aligned}
& \left\|\phi_{x}(t)\right\|^{2}+\int_{0}^{t}\left\|\phi_{x}(\tau)\right\|^{2} d \tau \\
& \quad \leq C\left\{\left\|\left(\phi_{0}, \psi_{0}\right)\right\|_{1}^{2}+e^{-c_{-} \beta}+N(T) \int_{0}^{t}\left[\left\|\phi_{x}(\tau)\right\|^{2}+\left\|\psi_{x}(\tau)\right\|_{1}^{2}\right] d \tau\right\} .
\end{aligned}
$$

Proof. From the equation (3.10), we have

$$
\frac{\mu \phi_{x t}}{V}+f(V) \phi_{x}=\psi_{t}-F
$$

Multiplying (4.20) by $\phi_{x}$ yields

$$
\left\{\frac{\mu}{2 V} \phi_{x}^{2}\right\}_{t}+\left(f(V)-\frac{h(V)}{2 V}\right) \phi_{x}^{2}=\psi_{t} \phi_{x}-F \phi_{x},
$$

where we used $\mu s V_{x}=V h(V)$. The first equation of (3.10) gives

$$
\begin{aligned}
\psi_{t} \phi_{x} & =\left\{\psi \phi_{x}\right\}_{t}-\psi \phi_{x t}=\left\{\psi \phi_{x}\right\}_{t}-\psi \psi_{x x} \\
& =\left\{\psi \phi_{x}\right\}_{t}-\left\{\psi \psi_{x}\right\}_{x}+\psi_{x}^{2} .
\end{aligned}
$$

Substituting (4.22) back into (4.21), and noting (4.10), we obtain

$$
\left\{\frac{\mu}{2 V} \phi_{x}^{2}-\psi \phi_{x}\right\}_{t}+\left|p^{\prime}\left(v_{+}\right)\right| \phi_{x}^{2} \leqq \psi_{x}^{2}-\left\{\psi \psi_{x}\right\}_{x}-F \phi_{x} .
$$


Integrating (4.23) over $[0, \infty) \times[0, t]$ and using $v_{-} \leqq V \leqq v_{+}$and the inequalities

$$
\begin{aligned}
\int_{0}^{\infty}\left|\psi_{0} \phi_{0, x}\right| d x & \leqq \frac{1}{2}\left(\left\|\psi_{0}\right\|^{2}+\left\|\phi_{0, x}\right\|^{2}\right), \\
\int_{0}^{\infty}\left|\psi \phi_{x}\right| d x & \leqq \frac{\mu}{4 v_{+}}\left\|\phi_{x}(t)\right\|^{2}+\frac{v_{+}}{\mu}\|\psi(t)\|^{2},
\end{aligned}
$$

we have

$$
\begin{aligned}
& \frac{\mu}{4 v_{+}}\left\|\phi_{x}(t)\right\|^{2}+\left|p^{\prime}\left(v_{+}\right)\right| \int_{0}^{t}\left\|\phi_{x}(\tau)\right\|^{2} d \tau \\
& \leqq \frac{\mu}{2 v_{-}}\left\|\phi_{0, x}\right\|^{2}+\frac{1}{2}\left\|\psi_{0}\right\|^{2}+\frac{1}{2}\left\|\phi_{0, x}\right\|^{2}+\left|\int_{0}^{t} \psi \psi_{x}\right|_{x=0} d \tau \mid+\frac{v_{+}}{\mu}\|\psi(t)\|^{2} \\
&+\int_{0}^{t}\left\|\psi_{x}(\tau)\right\|^{2} d \tau+\int_{0}^{t} \int_{0}^{\infty}\left|F \phi_{x}\right| d x d \tau .
\end{aligned}
$$

Applying the estimate (4.1) for the boundary and the basic estimate (4.12) to this inequality yields the estimate (4.19).

Lemma 4.5. For $t \in[0, T]$,

$$
\begin{aligned}
& \left\|\psi_{x}(t)\right\|^{2}+\int_{0}^{t}\left\|\psi_{x x}(\tau)\right\|^{2} d \tau \\
& \quad \leqq C\left\{\left\|\left(\phi_{0}, \psi_{0}\right)\right\|_{1}^{2}+e^{-c_{-} \beta}+N(T) \int_{0}^{t}\left[\left\|\phi_{x}(\tau)\right\|^{2}+\left\|\psi_{x}(\tau)\right\|_{1}^{2}\right] d \tau\right\} .
\end{aligned}
$$

Proof. Multiplying the second equation of (3.10) by $-\psi_{x x}$ gives

$$
\frac{1}{2}\left\{\psi_{x}^{2}\right\}_{t}-\left\{\psi_{x} \psi_{t}\right\}_{x}+f(V) \phi_{x} \psi_{x x}+\frac{\mu}{V} \psi_{x x}^{2}=-F \psi_{x x}
$$

Inequality (4.9) and the Cauchy inequality yield

$$
\left|f(V) \phi_{x} \psi_{x x}\right| \leqq \frac{\mu}{2 v_{+}} \psi_{x x}^{2}+\frac{c_{0}^{2} v_{+}}{2 \mu} \phi_{x}^{2},
$$

and (3.14) and the Cauchy inequality yield

$$
\left|-F \psi_{x x}\right| \leqq C\left(\left|\phi_{x}\right|^{2}+\left|\phi_{x}\right|\left|\psi_{x x}\right|\right)\left|\psi_{x x}\right| \leqq C\left|\phi_{x}\right|\left(\left|\phi_{x}\right|^{2}+\left|\psi_{x x}\right|^{2}\right) .
$$

Substituting (4.26), (4.27) and $\mu / V \geqq \mu / v_{+}$into (4.25), we have

$$
\frac{1}{2}\left\{\psi_{x}^{2}\right\}_{t}-\left\{\psi_{x} \psi_{t}\right\}_{x}+\frac{\mu}{2 v_{+}} \psi_{x x}^{2} \leqq \frac{c_{0}^{2} v_{+}}{2 \mu} \phi_{x}^{2}+C\left|\phi_{x}\right|\left(\left|\phi_{x}\right|^{2}+\left|\psi_{x x}\right|^{2}\right) .
$$

Integrating (4.28) over $[0, \infty) \times[0, t]$, and making use of the estimate (4.2) for the boundary and Lemmas 4.3 and 4.4, we have (4.24). 
Lemma 4.6. For $t \in[0, T]$,

$$
\begin{aligned}
\left\|\phi_{x x}(t)\right\|^{2} & +\int_{0}^{t}\left\|\phi_{x x}(\tau)\right\|^{2} d \tau \\
\leqq & C\left\{\left\|\left(\phi_{0}, \psi_{0}\right)\right\|_{2}^{2}+e^{-c_{-} \beta}+N(T) \int_{0}^{t}\left\|\phi_{x}(\tau)\right\|^{2} d \tau\right. \\
& \left.+N(T) \int_{0}^{t}\left\|\psi_{x}(\tau)\right\|_{1}^{2} d \tau+\int_{0}^{t}\left\|F_{x}(\tau)\right\|^{2} d \tau\right\} .
\end{aligned}
$$

Proof. Differentiating (4.20) with respect to $x$ and multiplying the derivative by $\phi_{x x}$, we have

$$
\begin{aligned}
& \left\{\frac{\mu}{2 V} \phi_{x x}^{2}\right\}_{t}+\left(f(V)-\frac{h(V)}{2 V}\right) \phi_{x x}^{2}+\frac{\mu V_{x}}{V^{2}} \phi_{x t} \phi_{x x}+f(V)_{x} \phi_{x} \phi_{x x} \\
& =\psi_{x t} \phi_{x x}-F_{x} \phi_{x x},
\end{aligned}
$$

where we used $\mu s V_{x}=V h(V)$. By the first equation of (3.10) and the Cauchy inequality, we have

$$
\begin{gathered}
\left|\frac{\mu V_{x}}{V^{2}} \phi_{x t} \phi_{x x}\right|=\left|\frac{\mu V_{x}}{V^{2}} \psi_{x x} \phi_{x x}\right| \leqq \frac{1}{4}\left|p^{\prime}\left(v_{+}\right)\right| \phi_{x x}^{2}+C\left|\psi_{x x}\right|^{2}, \\
\left|f(V)_{x} \phi_{x} \phi_{x x}\right| \leqq \frac{1}{4}\left|p^{\prime}\left(v_{+}\right)\right| \phi_{x x}^{2}+C\left|\phi_{x}\right|^{2}, \\
\left|F_{x} \phi_{x x}\right| \leqq \frac{1}{4}\left|p^{\prime}\left(v_{+}\right)\right| \phi_{x x}^{2}+C\left|F_{x}\right|^{2}, \\
\psi_{x t} \phi_{x x}=\left\{\psi_{x} \phi_{x x}\right\}_{t}-\psi_{x} \phi_{x x t}=\left\{\psi_{x} \phi_{x x}\right\}_{t}-\psi_{x} \psi_{x x x} \\
=\left\{\psi_{x} \phi_{x x}\right\}_{t}-\left\{\psi_{x} \psi_{x x}\right\}_{x}+\psi_{x x}^{2} .
\end{gathered}
$$

Substituting (4.31)-(4.34) into (4.30), integrating it over $[0, \infty) \times[0, t]$, and making use of (4.10), we then have

$$
\begin{aligned}
\left\|\phi_{x x}(t)\right\|^{2}+\int_{0}^{t}\left\|\phi_{x x}(\tau)\right\|^{2} d \tau \leqq & C\left\{\left\|\left(\phi_{0}, \psi_{0}\right)\right\|_{2}^{2}+e^{-c_{-} \beta}\right. \\
& \left.+\int_{0}^{t}\left(\left\|\phi_{x}(\tau)\right\|^{2}+\left\|\psi_{x}(\tau)\right\|_{1}^{2}+\left\|F_{x}(\tau)\right\|^{2}\right) d \tau\right\},
\end{aligned}
$$

which implies (4.29) by Lemmas 4.3-4.5, that is, we used the fact

$$
\begin{aligned}
\int_{0}^{t}\left(\left\|\phi_{x}(\tau)\right\|^{2}+\left\|\psi_{x}(\tau)\right\|_{1}^{2}\right) d \tau \leqq & C\left\{\left\|\left(\phi_{0}, \psi_{0}\right)\right\|_{2}^{2}+e^{-c_{-} \beta}\right. \\
& \left.+N(T) \int_{0}^{t}\left(\left\|\phi_{x}(\tau)\right\|^{2}+\left\|\psi_{x}(\tau)\right\|_{1}^{2}\right) d \tau\right\} .
\end{aligned}
$$

Thus, the proof is complete. 
Lemma 4.7. For $t \in[0, T]$,

$$
\begin{aligned}
& \left\|\psi_{x x}(t)\right\|^{2}+\int_{0}^{t}\left\|\psi_{x x x}(\tau)\right\|^{2} d \tau \\
& \leqq \\
& \quad C\left\{\left\|\left(\phi_{0}, \psi_{0}\right)\right\|_{2}^{2}+e^{-c_{-} \beta}+N(T) \int_{0}^{t}\left(\left\|\phi_{x}(\tau)\right\|_{1}^{2}+\left\|\psi_{x}(\tau)\right\|_{1}^{2}\right) d \tau\right. \\
& \left.\quad+\int_{0}^{t}\left\|F_{x}(\tau)\right\|^{2} d \tau\right\} .
\end{aligned}
$$

Proof. As in the proofs of Lemmas 4.5 and 4.6, we differentiate the second equation of (3.10) with respect to $x$, multiply the derivative by $-\psi_{x x x}$, integrate the resulting equality over $[0, \infty) \times[0, t]$, and make use of (4.10), Lemmas 4.1, 4.3-4.6, to prove (4.35). The details are omitted here.

Proof of Proposition 3.4. Combining Lemmas 4.3-4.7, we have

$$
\begin{aligned}
& \|(\phi, \psi)(t)\|_{2}^{2}+\int_{0}^{t}\left[\left\|\phi_{x}(\tau)\right\|_{1}^{2}+\left\|\psi_{x}(\tau)\right\|_{2}^{2}\right] d \tau \\
& \leqq \\
& \quad C\left\{\left\|\left(\phi_{0}, \psi_{0}\right)\right\|_{2}^{2}+e^{-c_{-} \beta}+N(T) \int_{0}^{t}\left[\left\|\phi_{x}(\tau)\right\|^{2}+\left\|\psi_{x}(\tau)\right\|_{1}^{2}\right] d \tau\right. \\
& \left.\quad+\int_{0}^{t}\left\|F_{x}(\tau)\right\|^{2} d \tau\right\} .
\end{aligned}
$$

Using the Sobolev lemma, we have by (3.13) and (3.14) that

$$
\begin{aligned}
\left\|F_{x}\right\|^{2} & \leqq C \int_{0}^{\infty}\left(\phi_{x}^{4}+\phi_{x}^{2} \phi_{x x}^{2}+\psi_{x x}^{2} \phi_{x x}^{2}+\psi_{x x x}^{2} \phi_{x}^{2}+\phi_{x}^{2} \psi_{x x}^{2}\right) d x \\
& \leqq C\left[\sup _{x \in R_{+}} \phi_{x}^{2} \int_{0}^{\infty}\left(\phi_{x}^{2}+\phi_{x x}^{2}+\psi_{x x x}^{2}+\psi_{x x}^{2}\right) d x+\sup _{x \in R_{+}} \psi_{x x}^{2} \int_{0}^{\infty} \phi_{x x}^{2} d x\right] \\
& \leqq C\left[\left\|\phi_{x}\right\|_{1}^{2}\left(\left\|\phi_{x}\right\|^{2}+\left\|\psi_{x x x}\right\|^{2}\right)+\left\|\psi_{x x}\right\|_{1}^{2}\left\|\phi_{x x}\right\|^{2}\right] \\
& \leqq C\left\|\phi_{x}\right\|_{1}^{2}\left(\left\|\phi_{x}\right\|_{1}^{2}+\left\|\psi_{x}\right\|_{2}^{2}\right) \\
& \leqq C N(T)\left(\left\|\phi_{x}\right\|_{1}^{2}+\left\|\psi_{x}\right\|_{2}^{2}\right) .
\end{aligned}
$$

Substituting (4.37) into (4.36) yields

$$
\begin{aligned}
\|(\phi, \psi)(t)\|_{2}^{2}+ & (1-C N(T)) \int_{0}^{t}\left[\left\|\phi_{x}(\tau)\right\|_{1}^{2}+\left\|\psi_{x}(\tau)\right\|_{2}^{2}\right] d \tau \\
& \leqq C\left\{\left\|\left(\phi_{0}, \psi_{0}\right)\right\|_{2}^{2}+e^{-c_{-} \beta}\right\} .
\end{aligned}
$$

Hence, choosing $N(T)$ so small that $N(T) \leqq \min \left\{\delta_{0}, C^{-1}\right\}$, we can prove the a priori estimate (3.15). 
To prove (3.16), we differentiate the first equation of (3.10) with respect to $x$, multiply it by $\phi_{x}$, and integrate the resulting equality with respect to $x$, to obtain

$$
\frac{d}{d t}\left\|\phi_{x}(t)\right\|^{2}=2 \int_{0}^{\infty} \psi_{x x} \phi_{x} d x .
$$

Then, from (3.15) we get

$$
\begin{aligned}
\int_{0}^{t}\left|\frac{d}{d t}\left\|\phi_{x}(t)\right\|^{2}\right| & \leqq \int_{0}^{t}\left(\left\|\phi_{x}(\tau)\right\|^{2}+\left\|\psi_{x x}(\tau)\right\|^{2}\right) d \tau \\
& \leqq C\left\{\left\|\left(\phi_{0}, \psi_{0}\right)\right\|_{2}^{2}+e^{-c_{-} \beta}\right\} .
\end{aligned}
$$

Similarly, the second equation of (3.10) and the estimate (3.15) give us

$$
\int_{0}^{t}\left|\frac{d}{d t}\left\|\psi_{x}(t)\right\|^{2}\right| \leqq C\left\{\left\|\left(\phi_{0}, \psi_{0}\right)\right\|_{2}^{2}+e^{-c_{-} \beta}\right\} .
$$

Thus, (4.39) and (4.40) imply the a priori estimate (3.16).

Acknowledgements. The work of M. MEI was supported in part by the JSPS Research Fellowship for Young Scientists and the Grand-in-aid No. P-96169 for JSPS from the Ministry of Education, Culture and Science of Japan.

\section{References}

1. Chern, I-L. \& MeI, M., Asymptotic stability of critical viscous shock waves for a degenerate hyperbolic viscous conservation laws, Commun. Partial Diff. Eqns., 23 (1998) 869-886.

2. Goodman, J., Nonlinear asymptotic stability of viscous shock profiles for conservation laws, Arch. Rational Mech. Anal., 95 (1986) 325-344.

3. Kawashima, S., Private communication.

4. Kawashima, S. \& Matsumura, A., Asymptotic stability of traveling wave solutions of system for one-dimensional gas motion, Commun. Math. Phys., 101 (1985) 97-127.

5. Kawashima, S. \& Matsumura, A., Stability of shock profiles in viscoelasticity with non-convex constitutive relations, Commun. Pure Appl. Math., 47 (1994) 1547-1569.

6. Liu, T.-P., Nonlinear stability of shock waves for viscous conservation laws, Mem. Amer. Math. Soc., 56 no. 328 (1985).

7. Liu, T.-P., Matsumura, A. \& Nishihara, K., Behavior of solutions for the Burgers equations with boundary corresponding to rarefaction waves, SIAM J. Math. Anal., 29 (1998) 293-308. Math. Anal., 29 (1998) 293-308.

8. Liu, T.-P. \& Nishinara, K., Asymptotic behavior for scalar viscous conservation laws with boundary effect, J. Diff. Eqns., 133 (1997) 296-320.

9. Liu, T.-P. \& YU, S.-H., Propagation of a stationary shock layer in the presence of a boundary, Arch. Rational Mech. Anal., 139 (1997) 57-82.

10. Liu, H.-L. \& WANG, J.-H., Nonlinear stability of viscous shock profiles for system of isentropic gas dynamics, unpublished.

11. Matsumura, A. \& MeI, M., Nonlinear stability of viscous shock profile for a non-convex system of viscoelasticity, Osaka J. Math., 34 (1997) 589-603.

12. Matsumura, A. \& Nishinara, K., On the stability of traveling wave solutions of a one-dimensional model system for compressible viscous gas, Japan J. Appl. Math., 2 (1985) 17-25. 
13. Matsumura, A. \& Nishihara, K., Asymptotics toward rarefaction wave for solution of the viscous p-system with boundary effect, to appear in Quart. Appl. Math.

14. MEI, M., Stability of traveling wave solutions for non-convex equations of barotropic viscous gas, Osaka J. Math., 34 (1997) 303-318.

15. Mei, M. \& Nishinara, K., Nonlinear stability of traveling waves for one dimensional viscous materials with nonconvex nonlinearity, Tokyo J. Math., 20 (1997) 241-264.

16. Nishinara, K., Stability of travelling waves with degenerate shock condition for onedimensional viscoelastic model, J. Diff. Eqns., 120 (1995) 304-318.

17. Pan, T., H.-X. Liu \& Nishihara, K., Asymptotic stability of the rarefaction wave of a one-dimensional model system for compressible viscous gas with boundary, to appear in Japan J. Indust. Appl. Math.

18. SzePESsY, A. \& XIN, Z.-P., Nonlinear stability of viscous shock waves, Arch. Rational Mech. Anal., 122 (1993) 53-104.

19. YU, S.-H.: Burgers equations on the quarter plane, preprint.

Department of Mathematics

Graduate School of Science Osaka University

Toyonaka 560-0043, Japan

e-mail: akitaka@math.sci.osaka-u.ac.jp

and

Department of Computational Science

Faculty of Science

Kanazawa University

Kanazawa 920-1192, Japan

e-mail: mei@kappa.s.kanazawa-u.ac.jp

(Accepted March 2, 1998) 